\title{
Serviços ecossistêmicos: conceitos e classificação
}

Os impactos provenientes das atividades antrópicas sobre os ecossistemas têm causado grandes preocupações quanto à quantidade, qualidade e disponibilidade dos recursos naturais, recursos estes imprescindíveis para a vida e o bem estar humano. Desta forma os ecossistemas ficam cada vez mais susceptível bem como os bens e serviços ofertados por eles. Diante disso o presente trabalho teve como objetivo apresentar as principais discussões sobre serviços ecossistêmicos tendo sido abordado conceitos de serviços ecossistêmicos, classificação e mapeamento de serviços ecossistêmicos.

Palavras-chave: Ecossistema; Meio Ambiente; Funções Ecossistêmicas; Mapeamento.

\section{Ecosystem services: concepts and classification}

The impacts from anthropic activities on ecosystems have raised major concerns about the quantity, quality and availability of natural resources, which are essentia resources for human life and well-being. In this way the ecosystems are increasingly susceptible as well as the goods and services offered by them. Therefore, the present work had as main objective to present the main discussions on ecosystem services, having addressed the concepts of ecosystem services, classification and mapping of ecosystem services.

Keywords: Ecosystem; Environment; Ecosystem Functions; Mapping.

Topic: Desenvolvimento, Sustentabilidade e Meio Ambiente

Reviewed anonymously in the process of blind peer.
Received: $10 / 04 / 2018$

Approved: 24/05/2018
Aldair dos Santos Gomes

Universidade Federal de Campina Grande, Brasil

http://lattes.cnpq.br/5584225078288019

http://orcid.org/0000-0001-9235-3337

aldairbiologo@gmail.com

José Dantas Neto

Universidade Federal de Campina Grande, Brasil

http://lattes.cnpq.br/9137226205129315

http://orcid.org/0000-0003-0798-6717

zedantas1955@gmail.com

Viviane Farias Silva

Universidade Federal de Campina Grande, Brasil

http://lattes.cnpq.br/5011520274887172

http://orcid.org/0000-0002-5891-0328

flordeformosur@hotmail.com

\section{Referencing this:}

GOMES, A. S.; DANTAS NETO, J.; SILVA, V. F.. Serviços ecossistêmicos: conceitos e classificação. Revista Ibero Americana de Ciências Ambientais, v.9, n.4, p.12-23, 2018. DOI:

http://doi.org/10.6008/CBPC2179-6858.2018.004.0002 


\section{INTRODUÇÃO}

A percepção acerca dos problemas ambientais surge como uma questão importante a respeito do crescimento material e econômico bem como da qualidade de vida. A qualidade de vida para alguns é alcançada a partir da limitação da produção de bens materiais, já para outros a qualidade de vida está intimamente ligada à abundância de bens materiais o que resulta na necessidade de aumento da produção consequentemente exercendo uma pressão maior sobre os recursos naturais (MATOS, 2005).

Os impactos gerados pela ação antropogênica nos diversos sistemas biológicos é crescente produzindo desta forma graves consequência em rios e lagos. Segundo Tundisi (1999) tem-se constatado que as águas se degradam devido às inúmeras atividades humanas que tem sido desenvolvidas intensamente em suas bacias hidrográficas.

Ao analisar a atual crise social, econômica e ambiental, é de fundamental importância compreender a influência das ações econômicas na dinâmica dos ecossistemas. Porém é primordial que se entenda as funções ecológicas, que posteriormente se traduzem em bens e serviços ecossistêmicos, os quais são relevantes para a existência e para a manutenção da vida, e o bem-estar humano, bem como para o desenvolvimento de atividades econômicas (NICHOLSON et al., 2009; DERISSEN et al., 2013; HAILS et al., 2013). É oportuno destacar que ecossocioeconomia corresponde a perspectiva de um modelo de desenvolvimento onde o crescimento econômico, o aumento igualitário do bem-estar social e a preservação ambiental são indissociáveis (SACHS, 2007).

Com a finalidade de tornar estas relações mais perceptíveis e quantificáveis, fazendo com que as questões de influência e dependência possam ser melhor manejadas, apresenta-se a ferramenta de valoração ambiental (BEDER, 2011; GÓMEZ-BAGGETHUN et al., 2011). Esta ferramenta, possibilita a identificação de valores econômicos único cálculo proposto pela Economia Ambiental (ELLIOT, 2005; NOBRE et al., 2002), mas também ecológicos e socioculturais tal ampliação foi proposta pela Economia Ecológica (COSTANZA, 2000; MARTíNEZ-ALIER et al., 1998) dos recursos naturais. Devidamente mensurado, tais 'valores', que podem apresentar-se de forma quantitativa e qualitativa, poderão ser utilizados nas tomadas de decisão que envolvem, dentre de várias questões, a temática ambiental (AMAZONAS, 2009; COSTANZA, 2003).

A ideia de valorar os serviços ecossistêmicos é muito discutida porém ainda muito criticada principalmente quando se trata de questões intangíveis, ou seja, quando não estão passiveis da estipulação de um valor monetário, como a vida, o ar que respiramos ou regiões sagradas. Porém, fazemos isso todos os dias e a todo o momento. Quando é decidido construir um determinado empreendimento, uma rodovia, criar um parque nacional ou permitir a visitação de uma praia, é perceptível a ponderação da importância de inúmeros elementos de acordo com o objetivo. De certo modo além de estar valorando serviços ecossistêmicos há o questões de outras ordens, como mobilidade, oferta de empregos e entretenimento. $O$ ato de possibilitar todo este processo de ponderação e denominá-lo de valoração é somente tornar mais evidente o que já é feito corriqueiramente. É deixar mais claro, por uma perspectiva diferente, o que está 
sendo levado em conta para a tomada de decisão, bem como as importâncias relativas, quem está envolvido e quais são as perdas e ganhos (COSTANZA, 2003; KUMAR et al., 2007).

\section{METODOLOGIA}

\section{Funções ecossistêmicas}

A compreensão do funcionamento dos ecossistemas exige um esforço de mapeamento das funções ecossistêmicas, as quais podem ser definidas como as constantes interações existentes entre os elementos estruturais de um ecossistema, incluindo transferência de energia, ciclagem de nutrientes, regulação de gás, regulação climática e do ciclo da água (DALY et al., 2004). Essas funções, são consideradas um subconjunto dos processos ecológicos e das estruturas ecossistêmicas possibilitando uma verdadeira interação sistêmica no interior dos ecossistemas, constituindo um todo maior que a soma das partes individuais (De GROOT et al., 2002). O conceito de funções ecossistêmicas se torna relevante, pois é por meio deles que acontece a produção dos chamados serviços ecossistêmicos, que são os benefícios diretos e indiretos conseguidos pelo homem a partir dos ecossistemas. Dentre eles pode-se citar a provisão de alimentos, a regulação climática e do ciclo da água a formação do solo, etc. (DAILY, 1997; COSTANZA et al., 1997; GROOT et al., 2002; MEA, 2003). São, em última instância, fluxos de materiais, energia e informações resultantes dos ecossistemas naturais e cultivados que, combinados com os demais tipos capital (humano, manufaturado e social) produzem o bem-estar humano.

Geralmente, uma função ecossistêmica produz um determinado serviço ecossistêmico quando os processos naturais subjacentes estimulam uma série de benefícios direta ou indiretamente apropriáveis pelo ser humano, agregando a noção de utilidade antropocêntrica. Em outras palavras, uma função passa a ser considerada um serviço ecossistêmico quando ela apresenta possibilidade/potencial de ser utilizada para fins humanos (HUETING et al., 1998). Os processos (funções) e serviços ecossistêmicos nem sempre apresentam uma relação biunívoca, sendo que um único serviço ecossistêmico pode ser o produto de duas ou mais funções, ou uma única função pode gerar mais que um serviço ecossistêmico (COSTANZA et al., 1997; GROOT et al., 2002).

A reciprocidade das funções ecossistêmicas faz com que a análise de seus serviços requeira a compreensão das interconexões existentes entre os seus componentes, preservando a capacidade dinâmica dos ecossistemas em realizar seus serviços (LIMBURG et al., 1999). A ocorrência das funções e serviços ecossistêmicos pode se dar em várias escalas espaciais e temporais tornando sua análise uma missão ainda mais complexa. Além disso, embora todos os serviços ecossistêmicos sejam derivados de funções ecossistêmicas nem todos os processos produzem serviços ecossistêmicos. Alguns processos podem não ser úteis para os seres humanos, mas isso não prejudica sua importância, para o meio natural certamente terá utilidade (MONONEN et al., 2016).

A vida no planeta Terra está profundamente coadunada à contínua capacidade de provisão de serviços ecossistêmicos (SUKHDEV, 2008; MEA, 2005). A demanda humana por serviços ecossistêmicos vem 
crescendo rapidamente, ultrapassando em muitos casos a capacidade de os ecossistemas fornecê-los. Desta forma, faz-se necessário não somente o esforço de compreensão no sentido da dinâmica inerente aos elementos estruturais dos ecossistemas, mas também é de crucial relevância compreender quais são os mecanismos de interação entre os fatores de mudança dos ecossistemas e sua capacidade de geração dos serviços ecossistêmicos, como também seus impactos adversos sobre o bem-estar humano.

\section{DISCUSSÃO TEÓRICA}

\section{Conceito de serviços ecossistêmicos}

O conceito de serviços ecossistêmicos tornou-se imprescindível para facilitar a compreensão da maneira como o ser humano interage com o meio natural (THORSEN et al., 2014). O conceito de serviços ecossistêmicos é relativamente recente, tendo sido utilizado pela primeira vez no final da década de 1960 (KING, 1966; HELLIWELL, 1969), mas só em 1997 teve as atenções voltadas para se, devido as publicações de Gretchen Daily, Nature Services: Societal Dependence on Natural Ecosystem (DAILY, 1997), e de Robert Costanza, The value of the wold 's ecosystem services and natural capital (COSTANZA et al., 1997).

Em mais de três décadas, cresceu consideravelmente o número de publicações focadas nesta temática surgindo desta forma inúmeros trabalhos que discutem o conceito objetivando torna-lo mais claro e coeso (FISHER et al., 2009). Mesmo diante da enorme quantidade de trabalhos publicados sobre serviços ecossistêmicos, o conceito continua em evolução encontrando-se na literatura diversas definições. As diversas definições utilizadas apontam que embora exista uma acentuada semelhança sobre a ideia central do que seja serviços ecossistêmicos, não existe uma padronização do conceito sendo aplicados vários e distintos conceitos, a depender da origem ecológica ou econômica da abordagem (OJEA et al., 2012; HÄYHÄ et al., 2014). Sendo assim, independente da definição em se, a conceituação do termo serviços ecossistêmicos apresenta conjectura consensual que permite um maior entendimento do mesmo.

Daily (1997) definiu serviços ecossistêmicos como sendo as condições e os processos a partir dos quais os ecossistemas naturais, e as espécies que os constituem, sustentam e permitem a vida humana. Para Daily, os serviços ecossistêmicos estão correlacionados com as condições e processos e demostram uma interação entre a ecologia e o bem-estar humano. A definição de Daily já apresentava uma perspectiva econômica na medida em que o conceito proposto se converge no bem-estar humano.

Costanza et al. (1997) evidencia pela primeira vez a estimativa do valor econômico de vários serviços ecossistêmicos em diversos biomas do mundo, definindo-os como 'os benefícios que as sociedades obtêm, direta ou indiretamente, das funções dos ecossistemas'. Mais adiante, a iniciativa Millennium Ecosystem Assessment (ALCAMO et al., 2003) utilizando como base os trabalhos desenvolvidos por (COSTANZA et al., 1997; DAILY, 1997), simplifica o conceito e define serviços ecossistêmicos como sendo 'os benefícios que as sociedades obtêm dos ecossistemas'.

Diversos autores, como Boyd et al. (2007), Bateman et al. (2011) e Haines-Young et al. (2013), propõem uma definição mais prática baseada numa perspectiva econômica inserindo no conceito de serviços 
ecossistêmicos somente os produtos finais da natureza diretamente consumidos ou utilizados para produzir bem-estar na sociedade. Para estes autores, serviços ecossistêmicos são os elementos da natureza diretamente utilizados ou consumidos para a produção de bem-estar humano. Correspondem a serviços finais na medida em que são as saídas dos ecossistemas. Tal abordagem faz com que os serviços ecossistêmicos possam ser contabilizados, avaliados e reconhecidos pelos mercados e agentes envolvidos na tomada de decisão, sem correr o risco da duplicidade durante a contabilização (BOYD et al., 2007; BATEMAN et al., 2011).

Baseando-se na abordagem de Boyd et al. (2007), Fisher et al. (2009) definem serviços ecossistêmicos como sendo os elementos dos ecossistemas utilizados, ativa ou passivamente, para produzir bem-estar humano. Contrariando Boyd et al. (2007), Fisher et al. (2009) consideram que os processos e as funções dos ecossistemas podem ser considerados serviços ecossistêmicos se forem consumidos ou utilizados, direta ou indiretamente, pelo ser humano. Portanto, existe serviço sempre que existir benefício para a sociedade.

O conceito de serviços ecossistêmicos é, no entanto totalmente antropocêntrico e é resultante de uma visão utilitarista da natureza. Este aspecto, se por um lado provoca críticas porque a natureza não é valorizada pelo seu valor intrínseco mas sim pelo seu valor de utilidade, por outro torna a utilização do conceito de serviços ecossistêmicos atraente e complexa, na medida em que ajuda a relatar as diferentes e complexas formas como as sociedades humanas estão intimamente ligadas e dependentes da natureza (HAINES-YOUNG et al., 2013).

\section{Classificação dos serviços ecossistêmicos}

A classificação dos serviços ecossistêmicos é uma tarefa complexa e técnica. Tal complexidade ocorre por vários fatores dentre eles pode-se citar a inexistência de um conceito único, preciso disseminado, capaz de captar toda variedade de formas pelas quais os ecossistemas sustentam a vida humana e contribuem para o bem-estar humano, outro fator agravante é a existência de uma ampla gama de propósitos ou aplicações com diferentes requisitos em termos de níveis de resolução espacial e temática (GROOT et al., 2010; HAINESYOUNG et al., 2013).

A classificação dos serviços ecossistêmicos tem evoluído, disponibilizando na literatura diversas propostas de sistemas de classificação (p. ex. COSTANZA et al., 1997; ALCAMO et al., 2003; FISHER et al., 2009; GROOT et al., 2010; HAINES-YOUNG et al., 2013; VALLÉS-PLANELLS et al., 2014). Diante destes fatos, a categorização dos serviços ecossistêmicos tentou tornar mais compreensíveis seus conceitos e identificar a sua real importância para o bem-estar humano perante a complexidade desta relação. Dentre as categorizações mais difundidas, destaca-se a da Avaliação do Milênio (AM, 2005) como a mais citada. Nela os serviços ecossistêmicos estão agrupados em quatro categorias: de provisão, de regulação, culturais e de apoio, como mostra a Tabela 1.

Os serviços de provisão são aqueles com capacidade de fornecer bens, como água potável para o consumo humano e/ou para a dessedentação de animais de produção e nativos, alimentos (frutos, sementes, 
raízes, mel, caça, pescado etc.), matéria-prima para geração de energia, fitofármacos, entre outros (COSTANZA et al., 1997; GROOT et al., 2002; MEA, 2005; TALLIS et al., 2013).

Tabela 1: Classificação dos serviços ecossistêmicos segundo a Avaliação do Milênio.

\begin{tabular}{|l|l|l|}
\hline Serviços Provisão & Serviços de Regulação & Serviços Culturais \\
Produtos obtidos & Benefícios obtidos da regulação dos processos dos & $\begin{array}{l}\text { Benefícios intangíveis obtidos } \\
\text { ecossistemas dos }\end{array}$ \\
do Ecossistema & ecossistemas & - Espirituais e religiosos \\
- Alimento & - Regulação do clima & - Recreativos e ecoturísticos \\
- Água doce & - Regulação de enfermidades & - Estéticos/Inspiradores \\
- Combustível & - Regulação da água & - Educacionais \\
- Fibras & - Purificação da água & \\
- Bioquímicos & \multicolumn{2}{|l|}{} \\
\hline $\begin{array}{l}\text { Serviços de Apoio } \\
\text { Serviços necessários para a produção de todos os outros serviços dos ecossistemas }\end{array}$ \\
- Formação do solo \\
\hline
\end{tabular}

Fonte: Adaptado do (MEA, 2005).

Os serviços reguladores correspondem a processos ecossistêmicos que regulam as condições ambientais essenciais para manter a vida, exemplo: diluição de águas residuais, controle de enchentes e de erosão, regulação do clima, purificação da atmosfera, decomposição de resíduos orgânicos e controle de pragas e de doenças. Os serviços de suporte são processos ecossistêmicos fundamentais para a existência de outros serviços fornecidos pela natureza, como: serviço de habitat, que corresponde ao espaço adequado para a sobrevivência de plantas e de animais selvagens, auxiliando na manutenção da diversidade biológica, funcional e genética. Os serviços culturais compreendem os benefícios oferecidos pela dinâmica ecológica com fins educacionais, recreativos, espirituais e beleza cênica (COSTANZA et al., 1997; GROOT et al., 2002; MEA, 2005; TALLIS et al., 2013).

As áreas urbanas disponibilizam de uma capacidade reduzida para manter e produzir bens e serviços oriundos da natureza, desta forma estão em constante dependência da integridade de serviços ecossistêmicos de áreas rurais para sustentar o crescimento econômico, a dinâmica ecológica e a qualidade de vida dos cidadãos (HAILS et al., 2013). Ainda é pertinente destacar que estudos sobre serviços ecossistêmicos demonstram os visíveis esforços da comunidade científica em pesquisar métodos para internalizar o que antes era considerado externalidade (dimensão ambiental) nas decisões políticas e para o sistema mercantil (GROOT et al., 2012; HAILS et al., 2013; WILLEMEN et al., 2013). Desta maneira, a perspectiva de serviços ecossistêmicos pode se configurar como um dos instrumentos facilitadores do processo de transformação da sociedade vigente (COSTANZA et al., 2014; HÄYÄ et al., 2015), sendo capaz auxiliar a promoção de sustentabilidade entre as relações humanas e o meio ambiente (ABSON et al., 2014; LUEDERITZ et al., 2015).

\section{Mapeamento de serviços ecossistêmicos}

O crescimento exponencial pelo interesse em pesquisas sobre serviços ecossistêmicos expôs de forma explicita a necessidade crescente de demonstra-los através do mapeamento, desta forma os estudos que abordam está temática vem registrando um aumento bastante significativo (EGOH et al., 2012; MAES et al., 2013). O mapeamento dos serviços ecossistêmicos constituem-se em colocar a capacidade, fluxo ou 
benefício dos mesmos em um mapa de forma clara e abrangente (MAES et al., 2013). Segundo Madureira et al. (2013) a capacidade corresponde a eficiência do ecossistema em fornecer o serviço (stock); sendo assim o fluxo seria a quantidade que é disponibilizada em um determinado intervalo de espaço e tempo, o benefício está relacionado com a conquista de valor do lado da oferta ou da procura, o resultado econômico da venda de uma certa quantidade do serviço ou o bem-estar resultante da provisão.

O mapeamento dos serviços ecossistêmicos é considerado uma ferramenta de grande utilidade, portanto é primordial que a mesma seja elaborada de forma clara e coesa, possibilitando desta maneira a identificação e priorização de problemas, sinergias e trade-offs entre as diferenças dos serviços ecossistêmicos e entre os serviços ecossistêmicos e a biodiversidade (MAES et al., 2013; HÄYHÄ et al., 2014). A elaboração dos mapas simplifica a comunicação entre as partes interessadas, devido a possibilidade da visualização dada a capacidade dos ecossistemas em produzir serviços. Sendo assim, o mapeamento pode contribuir para a identificação, planejamento e gestão de áreas de conservação e consequentemente, dos serviços ecossistêmicos disponíveis e auxiliar na recuperação de serviços importantes que deixaram de existir devido ao uso inadequado (MAES et al., 2013).

A necessidade de mapeamento tornou primordial o desenvolvimento de indicadores robustos que permitam mapear e modelar os serviços de ecossistema. Na revisão de literatura efetuada por Egoh et al. (2012), relativa ao período de 1997 a 2011, foram identificados os indicadores utilizados com maior frequência para mapear e modelar serviços do ecossistema.

Usando como elemento norteador a classificação de serviços ecossistêmicos proposta pela abordagem The Economics of Ecosystems and Biodiversity (TEEB), Kreuter et al. (2001) identificaram 67 estudos que recorreram ao mapeamento, os serviços de regulação e os serviços de aprovisionamento foram os serviços que receberam maior atenção dos especialistas, vindo em seguida os serviços culturais e, por último, os serviços de suporte. Desta forma foi identificado uma vasta variedade de indicadores utilizados para mapear os serviços de regulação e culturais, no entanto para os serviços de aprovisionamento e suporte os autores identificaram uma diversidade de indicadores bastante inferior se comparada aos demais serviços (RODRIGUES, 2015).

Atualmente está disponível inúmeras estratégias metodológicas para quantificar e mapear os serviços ecossistêmicos. Eigenbrod et al. (2010) dividiram as metodologias de mapeamento de serviços ecossistêmicos entre as que utilizam alguns dados primários da área de estudo e as que recorrem somente a indicadores proxy. A revisão realizada por Egoh et al. (2012) identifica as metodologias mais utilizadas para o mapeamento de serviços ecossistêmicos, para isto dividiram em três grupos: I) coleta de dados obtidos a partir de observações diretas; II) método proxy (indiretos) onde um único indicador, ou indicadores combinados, são utilizados para definir os serviços ecossistêmicos; III) modelos baseados em processos que utilizam indicadores como variáveis de uma equação.

Segundo Egoh et al. (2012) a maioria dos estudos recorre a métodos proxy para quantificar e mapear os serviços ecossistêmicos. Um indicador proxy é uma medida substituta utilizada para extrair conhecimento de uma determinada área de interesse quando não é possível mensurar o que se pretende de forma direta. 
A ausência de dados é o maior desafio para a obtenção do sucesso na avaliação dos serviços (Madureira et al., 2013) e por este motivo acaba os métodos proxy sendo utilizados predominantemente como ferramenta de mapeamento e quantificação de serviços ecossistêmicos (MAES et al., 2013). Devido a utilização de diversos métodos proxy em serviços de regulação, ressalva-se que pode ocorrer diferenças bastante significativas na quantificação e consequentemente, na valoração monetária dos serviços ecossistêmicos.

Os dados diretos são habitualmente usados na quantificação de serviços de provisionamento enquanto os modelos são usados, sobretudo, para quantificar serviços de regulação, devido a sua complexidade e impossibilidade de ser descritos por métodos proxy (EGOH et al., 2012). Alguns modelos recentemente desenvolvidos para este efeito são o InVEST (Integrated Valuation of ESS and Trade-offs), o MIMES (The Multi-scale Integrated Models of Ecosystem Services) e o ARIES (Artificial Intelligence for Ecosystem Services).

Diante do exposto se faz necessário mais dados primários para mapear diretamente os estoques e os fluxos reais de serviços ecossistêmicos e desta forma possibilitar a validação dos modelos atuais de mapeamento de serviços ecossistêmicos. Ocorre que muitas vezes, os dados utilizados nos métodos proxy não foram gerados no contexto de serviços ecossistêmicos, porém estão sendo reutilizados corriqueiramente para mapear serviços ecossistêmicos devido à ausência de informações primárias. Por exemplo, as relações de área de espécies (SAR) são um proxy biogeográfico utilizados na literatura científica para estimar um mecanismo para a adição de espécies Eigenbrod et al. (2010), enquanto Nelson et al. (2009) utilizou pontuações de SAR para quantificar um serviço ecossistêmico de apoio da adequação do habitat de um ecossistema de água doce para espécies de vertebrados.

\section{Mapeamento participativo}

Segundo Brown et al. (2015) o mapeamento participativo é um método relativamente novo, devido ao mesmo disponibilizar de uma abordagem complementar o mapeamento baseado em especialistas. Os grupos de interesse identificam em mapas impressos ou digitais, os serviços disponíveis que os ecossistemas disponibilizam para o seu bem-estar, sendo também possível a mensuração do grau de importância de cada serviço disponível (BROWN et al., 2012; GARCÍA-NIETO et al., 2015; PALOMO et al., 2013; PALOMO et al., 2014).

A referida abordagem é importante principalmente para o mapeamento de serviços de provisão e culturais, por dois motivos primeiro por utilizar como base o conhecimento local dos participantes, e o segundo devido ao fato de serem caraterizados pela sua intangibilidade, desta forma estando claramente dependentes do relacionamento dos indivíduos com a natureza (BROWN et al., 2015; CHAN et al., 2012; FAGERHOLM et al., 2012; NAHUELHUAL et al., 2014). Para Brown et al. (2015), tais serviços podem ser mapeados em workshops pequenos e interativos, os referidos eventos possibilitam um aumento significativo do capital social, no entanto o poder inferencial dos dados mapeados para o apoio a tomada de decisão poderá ser reduzido. 
Este mapeamento demonstra as percepções dos agentes locais envolvidos, desta maneira se torna ainda mais importante o envolvimento dos grupos de interesse, devido ocorrer diferentes expressões e padrões durante a seleção e mapeamento dos serviços ecossistêmicos, possibilitando assim a partilha do conhecimento e o empoderamento dos agentes locais (FAGERHOLM et al., 2012; GARCÍA-NIETO et al., 2015). De acordo com Klain et al. (2012), esta metodologia pode ser aplicada para mensurar os valores monetários e não-monetários dos serviços, e também das pressões a que os serviços ecossistêmicos estão sujeito em uma determinada localidade.

Brown et al. (2014) identificaram quatro prioridades para o mapeamento colaborativo no sentindo de qualificar e tornar a metodologia mais consistente: a elevação do número de grupos de interesse, uma abordagem estratégica que possibilite a fidelidade dos dados, a melhoria do componente de participação que apresentara como consequência uma melhora na colaboração da comunidade e por último a avaliação da eficiência do mapeamento.

Portanto os métodos de mapeamento necessariamente devem ser priorizados e escolhidos de acordo com o objetivo e o nível de exigência do seu objetivo final (BROWN et al., 2015; SCHRÖTER et al., 2015). É preciso levar em consideração durante a escolha da metodologia a possibilidade da mesma de gerar o aumento do nível de aceitação da mesma dentro da sociedade, durante o período em que se torna relevante para os agentes locais (BROWN et al., 2015; WILLEMEN et al., 2015). O engajamento dos agentes locais no processo de mapeamento, bem como os problemas de imprecisão dos métodos e da cartografia e, a inconsistência das metodologias e indicadores são os maiores desafios a serem vencidos nesta temática do mapeamento dos serviços ecossistêmicos (CROSSMAN et al., 2013; HOU et al., 2013; MAES et al., 2016; MARTÍNEZ-HARMS et al., 2012; SCHÄGNER et al., 2013; WILLEMEN et al., 2015).

\section{CONCLUSÕES}

A partir dos estudo abordados foi possível perceber que apesar do crescimento exponencial de pesquisas sobre serviços ecossistêmicos ainda não existe um conceito fechado do que seja serviços ecossistêmicos bem como um modelo único de classificação dos serviços ecossistêmicos, no entanto é valido destacar que os conceitos e modelos de classificação existentes apresentam em seu eixo central um alto grau similaridade este fato possibilita uma aglutinação dos diversos conceitos e sistemas de classificação na perspectiva de qualificar os diversos entendimentos e chegar a um denominador comum.

Diante disso percebe-se a necessidade de estudos mais integrados no sentido de mapear e classificar os serviços ecossistêmicos, o esforço de mapeamento dos serviços resultar no aumento da possibilidade de conservação e ou preservação do mesmo, tal fato pode depender diretamente da metodologia utilizada para elaboração do mapeamento dos serviços ecossistêmicos.

\section{REFERÊNCIAS}

ABSON, D. J.; WEHRDEN, H. VON.; BAUMGÄRTNER, S.; FISCHER, J.; HANSPACH, J.; HÄRDTLE, W.; HEINRICHS, H.; KLEIN, A. M.; LANG, D. J.. Ecosystem service as a boundary object for sustainability. Ecological Economics, v.103, p.2937,2014 
ALCAMO, J. et al.. Ecosystems and human well-being: a framework for assessment. Millennium Ecosystem Assessment. Washington: Island Press, 2003.

AMAZONAS, M. C.. Valor ambiental em uma perspectiva heterodoxa institucional-ecológica. Economia e Sociedade, v.18, n.1(35), p.183-212, 2009.

BATEMAN, I. J.; MACE, G. M.; FEZZI, C.; ATKINSON, G.; TURNER, K.. Economic analysis for ecosystem service assessments. Environmental and Resource Economics, v.48, n.2, p.177-218, 2011.

BALNERA, P.; URIARTE, M.; ALMEIDA-LEÑERO, L.; ALTESOR, A.; DECLERCK, F.; GARDNER, T.; HALL, J.; LARA, A.; LATERRA, P.; PEÑA-CLAROS, M.; MATOS, D. M. S.; VOGL, A. L.; ROMERO-DUQUE, L. P.; ARREOLA, L. F.; CARO-BORRERO, A. P.; GALLEGO, F.; JAIN, M.; LITTLE, C.; XAVIER, R. O.; PARUELO, J. M.; PEINADO, J. E.; POORTER, L.; ASCARRUNZ, N.; CORREA, F.; CUNHA-SANTINO, M. B.; HERNÁNDEZSÁNCHEZ, A.; VALLEJOS, M.. Ecosystem services research in Latin America: the state of the art. Ecosystem Services, v.2, p.56-70, 2012.

BEDER, S.. Environmental economics and ecological economics: the contribution of interdisciplinarity to understanding, influence and effectiveness. Environmental Conservation, v.38, n.2, p.140-150, 2011.

BOYD, J.; BANZHAF, S.. What are ecosystem services?. The need for standardized environmental accounting units. Ecological Economics, v.63, p.616-626, 2007.

BROWN, G.; KYTTÄ, M.. Key issues and research priorities for public participation GIS (PPGIS): A synthesis based on empirical research. Applied Geography, v.46, p.126-136, 2014.

BROWN, G.; FAGERHOLM, N.. Empirical PPGIS/PGIS mapping of ecosystem services: A review and evaluation. Ecosystem Services, v.13, p.119-133, 2015.

CHAN, K. M. A.; SATTERFIELD, T.; GOLDSTEIN, J.. Rethinking ecosystem services to better address and navigate cultural values. Ecological Economics, v.74, p.8-18, 2012.

COSTANZA, R.; GROOT, R.; SUTTON, P.; PLOEG, S. VAN DER.; ANDERSON, S. J.; KUBISZEWSKI, J.; FARBER, S.; TURNER, R. K.. Changes in the global value of ecosystem services. Global Environmental Change, v.26, p.152-158, 2014.

COSTANZA, R.. Social goals and the valuation of ecosystem services. Ecosystems, v.3, p.4-10, 2000.

COSTANZA, R.. Social goals and the valuation of natural capital. Environmental Monitoring and Assessment, v.86, p.19-28, 2003.

COSTANZA, R.; D'ARGE, R.; GROOT, R.; FARBERK, S.; GRASSO M.; HANNON, B.; LIMBURG, K.; NAEEM, S.; O'NEILL, R. V.; PARUELO, J.; RASKIN, R. G.; CROSSMAN, N. D.; BURKHARD, B.; NEDKOV, S.; WILLEMEN, L.; PETZ, K.; PALOMO, I.; MAES, J.. A blueprint for mapping and modelling ecosystem services. Ecosystem Services, v.4, p.4-14, p.2013.

DALY, H. E.; FARLEY, J.. Ecological Economics: principles and applications. Washington: Island Press, 2004.
DAILY, G.. Nature's services: societal dependence on natural ecosystem. Washington: Island Press, 1997

DERISSEN, S.; LATACZ-LOHMANN, U.; What are PES?. A review of definitions and an extension. Ecosystem Services, v.6, p.12-15, 2013.

EGOH, B.; DRAKOU, E.G.; DUNBAR, M. B.; MAES, J.; WILLEMEN, L.. Indicators for mapping ecosystem services: a review. Joint Reserch Centre Scientific and Policy Reports. European Commission. 2012.

EIGENBROD, F.; ARMSWORTH, P. R.; ANDERSON, B. J.; HEINEMEYER, A.; SILLINGS, S.; ROY, D. B.; THOMAS, C. D.; GASTON, K. J.. The impact of proxy-based methods on mapping the distribution of ecosystem services. Journal of Applied Ecology, v.47, n.2, p.377-385, 2010.

ELLIOT, S. R.. Sustainability: an economic perspective. Resources Conservation and Recycling, v.44, p.263-277, 2005.

FAGERHOLM, N.; KÄYHKÖ, N.; NDUMBARO, F.; KHAMIS, M. Community stakeholders' knowledge in landscape assessments - Mapping indicators for landscape services. Ecological Indicators, v.18, n.80, p.421-433, 2012.

FISHER, B.; TURNER, R. K.; MORLING, P.. Defining and classifying ecosystem services for decision making. Ecological Economics, v.68, p.643-653, 2009.

GARCÍA-NIETO, A. P.; QUINTAS-SORIANO, C.; GARCÍALLORENTE, M.; PALOMO, I.; MONTES, C.; MARTÍN-LÓPEZ, B. Collaborative mapping of ecosystem services: The role of stakeholders' profiles. Ecosystem Services, v.13, p.141-152, 2015.

GÓMEZ-BAGGETHUN, E.; PÉREZ, M. R.. Economic valuation and the commodification of ecosystem service. Progress in Physical Geography, v.35, n.5, p.613-628, 2011.

GROOT, R. S.; WILSON, M. A.; BOUMANS, R. M. J.. A typology for the classification, description, and valuation of ecosystem functions, goods and services. Ecological Economics, v.41, p.393-408, 2002.

GROOT, R. S.; FISHER, B.; CHRISTIE, M.. Integrating the ecological and economic dimensions in biodiversity and ecosystem service valuation (Chapter 1). In: THE ECONOMICS OF ECOSYSTEMS AND BIODIVERSITY: ECOLOGICAL AND ECONOMIC FOUNDATIONS. Anais. London: Earthscan, 2010.

HAILS, R. S.; ORMEROD, S. J.. Ecological science for ecosystem services and the stewardship of natural capital. Journal of Applied Ecology, v.50, p.807-811, 2013.

HAINES-YOUNG, R.; POTSCHIN, M.. Common International Classification of Ecosystem Services (CICES): Consultation on Version 4, August-December 2012. EEA Framework Contract n. EEA/IEA/09/003. (2013).

HÄYHÄ, T.; FRANZESE, P. P.. Ecosystem services assessment: A review under an ecological-economic and systems perspective. Ecological Modelling, v.289, p.124-132, 2014. 
HÄYHÄ, T.; FRANZESE, P. P.; PALETTO, A.; FATH, B. D. Assessing, valuing, and mapping ecosystem services in Alpine forests. Ecosystem Services, v.14, p.12-23, 2015.

HELLIWELL, D. R.. Valuation of wildlife resources. Regional Studies, v.3, p.41-49, 1969.

HOU, Y.; BURKHARD, B.; MÜLLER, F.. Uncertainties in landscape analysis and ecosystem service assessment. Journal of Environmental Management, v.127, p.S117S131, p.2013.

HUETING, R.; REIJNDERS, L.; BOER, B.; LAMBOOY, J.; JANSEN, $H$.. The concept of environmental function and its valuation. Ecological Economics, v.25, p.31-35, 1998.

KING, R. T.. Wildlife and man. NY Conservationist, v.20, n.6, p.8-11, 1966.

KLAIN, S. C.; CHAN, K. M. A.. Navigating coastal values: Participatory mapping of ecosystem services for spatial planning. Ecological Economics, v.82, p.104-113, 2012.

KREUTER, U. P.; HARRIS, H. G.; MATLOCK, M.; LACEY, R. E.. Change in ecosystem service values in San Antonio area, Texas. Ecological Economics, v.39, p.333-346, 2001.

KUMAR, M.; KUMAR, P.. Valuation of ecosystem services: a psycho-cultural perspective. Ecological Economics, v.64, p.808-819, 2007.

LANG, D. L.; WAMSLER, C.; WEHRDEN, H.. A review of urban ecosystem services: six key challenges for future research. Ecosystem Services, v.14, p.98-112, 2015.

LIMBURG, K. E.; FOLKE, C.. The ecology of ecosystem services: introduction to the special issue. Ecological Economics, v.29, p.179-182, 1999.

LUEDERITZ, C.; BRINK, E.; GRALLA, F.; HERMELINGMEIER, V.; MEYER, M.; NIVEN, L.; PANZER, L.; PARTELOW, S.; RAU, A.; SASAKI,R.; ABSON, D. J.; LANG, D. L.; WAMSLER, C.; WEHRDEN, H.. A review of urban ecosystem services: six key challenges for future research. Ecosystem Services, v.14, p.98-112, 2015.

MAES, J. MAES, J.; TELLER, A.; ERHARD, M.; LIQUETE, C.; BRAAT, L.; BERRY, P.; EGOH, B.; PUYDARRIEUX, P.; FIORINA, C.; SANTOS, F.; PARACCHINI, ML.; KEUNE, H.; WITTMER, H.; HAUCK, J.; FIALA, I.; VERBURG, P. H.; CONDÉ, S.; SCHÄGNER, J. P.; SAN MIGUEL, J.; ESTREGUIL, C.; OSTERMANN, O.; BARREDO, J. I.; PEREIRA, H. M.; STOTT, A.; LAPORTE, V.; MEINER, A.; OLAH, B.; ROYO, G. E.; SPYROPOULOU, R.; PETERSEN, J. E.; MAGUIRE, C.; ZAL, N.; ACHILLEOS, E.; RUBIN, A.; LEDOUX, L.; BROWN, C.; RAES, C.; JACOBS, S.; VANDEWALLE M.; CONNOR, D.; BIDOGLIO, G.. Mapping and Assessment of Ecosystems and their Services: An analytical framework for ecosystem assessments under action 5 of the EU biodiversity strategy to 2020. Luxembourg: Publications Office of the European Union, 2013.

MAES, J.; CROSSMAN, N. D.; BURKHARD, B.. Mapping Ecosystem Services. In: POTSCHIN, M.; HAINES-YOUNG, R.; FISH, R.; TURNER, R. K.. Routledge Handbook of Ecosystem Services. London: Routlegde, 2016. p.188-204.
MADUREIRA, L.; MAGALHÃES, P.; SILVA, P. G.; MARINHO, C.; OLIVEIRA, R.. Economia dos serviços de ecossistema: Um guia para conhecer e valorizar serviços de agroecossistemas em áreas protegidas de montanha. Lisboa: Quercus Associação Nacional de Conservação da Natureza, 2013.

MARTÍNEZ-HARMS, M. J.; BALVANERA, P.. Methods for mapping ecosystem service supply: A review. International Journal of Biodiversity Science, Ecosystems Services and Management, v.8, n.1-2, p.17-25, 2012.

MARTíNEZ-ALIER, J.; MUNDAA, G.; O'NEILLB, J.. Weak comparability of values as a foundation for ecological economics. Ecological Economics, v.26, n.3, p.277-286, 1998.

MEA. Millennium Ecosystem Assessment. Ecosystems and human well-being: biodiversity synthesis. Washington: World Resources Institute, 2005. p.86.

MEA. Millennium Ecosystem Assessment. Ecosystems Ecosystem and Human Well-Being: a framework for assessment. Washington: Island Press, 2003.

MONONEN, L.; AUVINEN, A. P.; AHOKUMPU, A. L.; RÖNKA, M.; AARRAS, N.; TOLVANEN, G.; KAMPPINEN, M.; VIIRRET, E.; KUMPULA, T.; VIHERVAARA, P.. Nationalecosystem service indicators: measures of social-ecological sustainability. Ecol. Indic. v.1, p.26-37, 2016.

NAHUELHUAL, L.; CARMONA, A.; LATERRA, P.; BARRENA, J.; AGUAYO, M.. A mapping approach to assess intangible cultural ecosystem services: The case of agriculture heritage in Southern Chile. Ecological Indicators, v.40, p.90-101, 2014.

NICHOLSON, E.; MACE, G. M.; ARMSWORTH, P. R.; ATKINSON, G.; BUCKLE, S.; CLEMENTS, T.; EWERS, R. M.; FA, J. E.; GARDNER, T. A.; GIBBONS, J.; GRENYER, R.; METCALFE, R.; MOURATO, S.; MUÛLS, M.; OSBORN, D.; REUMAN, D. C.; WATSON, C.; MILNER-GULLAND, E. J.. Priority research areas for ecosystem services in a changing world. Journal of Applied Ecology, v.46, p.1139-1144, 2009.

NELSON, E.; MENDOZA, G.; REGETZ, J.; POLASKY, S.; TALLIS, H.; CAMERON, D. R.; CHAN, K. M. A.; DAILY, G. C.; GOLDSTEIN, J.; KAREIVA, P. M.; LONDSORF, E.; NAIDOO, R.; RICKETTS, T. H.; SHAW, M. R.. Modeling multiple ecosystem services, biodiversity conservation, commodity production, and tradeoffs at landscape scales. Frontiers in Ecology and the Environment, Washington, v.7, p.4-11, 2009.

OJEA, E.; MARTIN-ORTEGA, J.; CHIABAI, A.. Defining and classifying ecosystem services for economic valuation: the case of forest water services. Environmental Science \& Policy, v.19, n.20, p.1-15, 2012.

PALOMO, I.; MARTÍN-LÓPEZ, B.; ZORRILLA-MIRAS, P.; GARCÍA DEL AMO, D.; MONTES, C.. Deliberative mapping of ecosystem services within and around Doñana National Park. 2014.

PALOMO, I.; MARTÍN-LÓPEZ, B.; POTSCHIN, M.; HAINESYOUNG, R.; MONTES, C.. National Parks, buffer zones and surrounding lands: Mapping ecosystem service flows. Ecosystem Services, v.4, n.2005, p.104-116, 2013. 
RODRIGUES, A. P.. Quantificação, valoração e mapeamento de serviços de ecossistema na bacia superior do Rio Sabor (concelho de Bragança). Dissertação (Mestrado) - Instituto Politécnico de Bragança, Bragança, 2015.

SACHS, I.. Rumo à ecossocioeconomia: teoria e prática do desenvolvimento. São Paulo: Cortez, 2007.

SCHRÖTER, M.; REMME, R. P.; SUMARGA, E.; BARTON, D. N. HEIN, L.. Lessons learned for spatial modelling of ecosystem services in support of ecosystem accounting. Ecosystem Services, v.13, p.64-69, 2015

SCHÄGNER, J. P.; BRANDER, L.; MAES, J.; HARTJE, V.. Mapping ecosystem services' values: Current practice and future prospects. Ecosystem Services, v.4, p.33-46, 2013.

\section{SUKHDEV, P.. The Economics of Ecosystems and}

Biodiversity. Interim Report of the Convention on Biological Diversity. Cambridge: European Communities, 2008.

SUTTONKK, P.; VAN DEN BELT, M.. The value of the world's ecosystem services and natural capital. Nature, v.387, p.253260, 1997.

TALLIS, H. T.; RICKETTS, T.; GUERRY, A. D.; WOOD, S.A.; SHARP, R.; NELSON, E.; ENNAANAY, D.;WOLNY, S.; OLWERO,
N.; VIGERSTOL, K.; PENNINGTON, D.; MENDOZA, G.; AUKEMA, J.; FOSTER, J.; FORREST, J.; CAMERON, D.; ARKEMA, K.; LONSDORF, E.; KENNEDY, C.; VERUTES, G.; KIM, C. K.; GUANNEL, G.; PAPENFUS, M.; TOFT, J.; MARSIK, M.; BERNHARDT, J.; GRIFFIN, R.. User's Guide: integrated valuation of environmental services and tradeoffs. Stanford: The Natural Capital Project, 2013.

TUNDISI, J. G.. Limnologia no século XXI: perspectivas e desafios. In: CONFERÊNCIA DE ABERTURA DO VII CONGRESSO BRASILEIRO DE LIMNOLOGIA. Anais. Instituto Internacional de Ecologia, 1999. p.24.

TEEB K, P.. The Economics of Ecosystems and Biodiversity: Ecological and Economic Foundations. London: Earthscan. 2010.

VALLÉS-PLANELLS, M.; GALIANA, F.; VAN EETVELDE, V.. A classification of landscape services to support local landscape planning. Ecology and Society, v.19, n.1, p.44, 2014.

WILLEMEN, L.; BURKHARD, B.; CROSSMAN, N.; DRAKOU, E. G.; PALOMO, I.. Best practices for mapping ecosystem services. Ecosystem Services, v.13, p.1-184, 2015.

A CBPC - Companhia Brasileira de Produção Científica (CNPJ: 11.221.422/0001-03) detém os direitos materiais desta publicação. Os direitos referem-se à publicação do trabalho em qualquer parte do mundo, incluindo os direitos às renovações, expansões e disseminações da contribuição, bem como outros direitos subsidiários. Todos os trabalhos publicados eletronicamente poderão posteriormente ser publicados em coletâneas impressas sob coordenação da Sustenere Publishing, da Companhia Brasileira de Produção Científica e seus parceiros autorizados. Os (as) autores (as) preservam os direitos autorais, mas não têm permissão para a publicação da contribuição em outro meio, impresso ou digital, em português ou em tradução. 